\title{
Blood Outgrowth Endothelial Cells Reduce Hypoxia-Mediated Fibroblast to Myofibroblast Conversion by Decreasing Proangiogenic Cytokines
}

\author{
Evelyn C. Nieves Torres ${ }^{a}$ Binxia Yang ${ }^{a} \quad$ Akshaar Brahmbhatt $^{a}$ \\ Debabrata Mukhopadhyay ${ }^{b}$ Sanjay Misra ${ }^{a, b}$

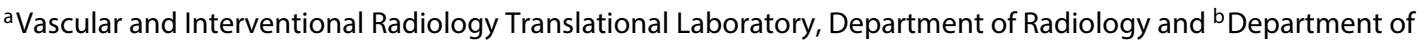 \\ Biochemistry and Molecular Biology, Mayo Clinic, Rochester, Minn., USA
}

\section{Key Words}

Blood outgrowth endothelial cells · Vascular endothelial growth factor A · Fibroblasts · Hypoxia

\begin{abstract}
Purpose: Hypoxic conditions cause fibroblasts to differentiate into alpha smooth-muscle cell actin (a -SMA)-positive cells, i.e. myofibroblasts. This process is a hallmark of venous neointimal hyperplasia ( $\mathrm{VNH}$ ) associated with hemodialysis vascular access. The purpose of this study was to determine if blood outgrowth endothelial cells (BOEC) may reduce the conversion of fibroblasts into myofibroblasts under hypoxic conditions, and to determine the potential mechanisms involved. Methods: An experimental model was used, in which fibroblasts and BOEC were subjected to hypoxia under contact and transwell conditions to determine if BOEC reduce the conversion of fibroblasts into myofibroblasts under hypoxic conditions. Gene expression under different conditions was performed. In addition, functional assays including cell proliferation and migration were determined. Results: This study demonstrates that contact needs to occur between BOEC and fibroblasts for the reduction of the hypoxiadriven conversion of fibroblasts into a-SMA. This is associated with a decrease in several proangiogenic genes includ-
\end{abstract}

ing vascular endothelial growth factor A, platelet-derived growth factor, fibroblast growth factor and matrix metalloproteinase 2 in fibroblasts in contact with BOEC when compared to fibroblasts alone. In addition, migration is significantly reduced while proliferation remains unchanged. Conclusion: This study helps provide rationale for using BOEC delivered to the adventitia of the outflow vein of hemodialysis vascular access to reduce $\mathrm{VNH}$.

(c) 2015 S. Karger AG, Basel

\section{Introduction}

In the next decade, it is estimated that the number of patients with end-stage renal disease who require chronic hemodialysis will double [1]. Today, in the USA, there are more than 400,000 patients who are on hemodialysis and they require a well-functioning hemodialysis vascular access for the clearance of uremic toxins. The preferred vascular access is the arteriovenous fistula (AVF), but its durability is limited by stenosis formation which is in part responsible for the poor patency rates of AVFs, estimated to be $62 \%$ at 1 year [2]. In order to maintain optimal functioning of hemodialysis AVFs and grafts, more than 1 billion USD are spent annually [1]. There-

\begin{tabular}{ll}
\hline KARGER & $\begin{array}{l}\text { ( ) 2015 S. Karger AG, Basel } \\
1018-1172 / 15 / 0516-0458 \$ 39.50 / 0 \quad \text { Karger }\end{array}$ \\
$\begin{array}{l}\text { E-Mail karger@karger.com } \\
\text { www.karger.com/jvr }\end{array}$ & $\begin{array}{l}\text { This is an Open Access article licensed under the terms of the } \\
\text { Creative Commons Attribution-NonCommercial 3.0 Un- } \\
\text { ported license (CC BY-NC) (www.karger.com/OA-license), } \\
\text { applicable to the online version of the article only. Distribu- } \\
\text { tion permitted for non-commercial purposes only. }\end{array}$
\end{tabular}

Prof. Sanjay Misra

Department of Radiology, Mayo Clinic

200 First Street SW

Rochester, MN 55905 (USA)

E-Mail misra.sanjay@ mayo.edu 
fore, novel therapies that could be developed to reduce AVF stenosis would be of tremendous benefit to patients with end-stage renal disease.

Patients with end-stage renal disease have an imbalance between endothelial and smooth-muscle progenitor cells; it has been hypothesized that this results in the increased incidence of cardiovascular complications accompanied by poor clinical outcomes [3, 4]. Blood outgrowth endothelial cells (BOEC) are a subset of circulating progenitor cells that possess an endothelial-cell phenotype and have the ability to reduce vascular injury [5]. A recent study from our laboratory demonstrated that BOEC transplanted to the adventitia of the outflow vein at the time of the creation of arteriovenous polytetrafluoroethylene grafts have reduced venous stenosis formation along with a decrease in hypoxia-inducible factor 1 alpha (HIF-1 $\alpha$ ) mean intima-to-media ratio when compared to controls [6]. Our other recent studies demonstrate that the hypoxia-mediated conversion of fibroblasts to myofibroblasts may play a role in venous neointimal hyperplasia $(\mathrm{VNH})$ and a reduced mean HIF-1 $\alpha$ level [7].

We demonstrated that inhibiting vascular endothelial growth factor A (VEGF-A), using a short-hairpin RNA inhibitor delivered to the adventitia of the outflow vein in a murine model of chronic kidney disease with an AVF, results in positive vascular remodeling [8]. In this study, we hypothesized that the vasa vasorum of the outflow vein of the AVF may become disrupted after surgical placement, which contributes to local hypoxia within the adventitia. Moreover, the localized hypoxia activates fibroblasts that convert into myofibroblasts, and subsequently triggers a cascade of events resulting in $\mathrm{VNH}$. Finally, reducing mRNA for VEGF-A in fibroblasts which are subjected to hypoxia results in a reduction of alpha smooth-muscle cell actin ( $\alpha$-SMA)-positive cells with a resultant decrease in matrix metalloproteinase 2 (MMP2), cellular proliferation and migration.

We hypothesized that BOEC may reduce the differentiation of fibroblasts into myofibroblasts under hypoxic conditions. The experiments outlined here were performed to determine the mechanism using a contact and transwell experimental design. We observed that a reduction in the conversion of fibroblasts into myofibroblasts occurs; this is accompanied by a reduction in several proangiogenic genes including VEGF-A, fibroblast growth factor-1 (FGF-1), platelet-derived growth factor (PDGF) and MMP-2. Each of these cytokines has been found to be associated with experimental animal models of hemodialysis vascular access failure or hemodialysis vascular access specimens removed from patients [9-14]. We speculate that adventitial delivery of BOEC to the AVF or graft at the time of creation could reduce $\mathrm{VNH}$, thus offering a novel therapeutic option.

\section{Materials and Methods}

\section{Isolation of $B O E C$}

Porcine BOEC were isolated according to the protocol used by our laboratory and described previously [6]. Briefly, under aseptic conditions, we removed $100 \mathrm{ml}$ of blood from the femoral artery and expanded it ex vivo as previously described, with some modifications [15]. Peripheral blood mononuclear cells were isolated using density gradient centrifugation with Ficoll-Paque Plus (Amersham Biosciences Corp., Piscataway, N.J., USA). The cell culture was maintained at $37^{\circ} \mathrm{C}, 5 \% \mathrm{CO}_{2}$ and $20 \% \mathrm{O}_{2}$. After 2 days, the media was changed and the nonadherent cells were removed. After satisfactory initial growth, the media was subsequently changed every 2-3 days. Typically, colonies of cells appeared that exhibited typical endothelial morphology including a cobblestone pattern; this occurred between day 15 and day 18. These colonies were passaged and expanded for an additional 2 weeks and the adherent cells were harvested by trypsinization. The late-outgrowth BOEC were characterized by means of a number of methods including staining and morphology as described previously [6]. All BOEC used in the experiments were from early passages with a maximal passage of 5 .

\section{Isolation of Fibroblasts}

Primary fibroblasts were isolated from the jugular vein of pigs as described elsewhere [16].

\section{Transwell Culture and Contact Experiments with BOEC and}

Fibroblasts

Fibroblasts and BOEC were isolated from pigs as described above and used for both transwell and contact experiments. For both experiments, 400,000 BOEC and 100,000 fibroblasts were used. The transwell experiments were performed with BOEC only in the upper chamber and fibroblasts only in the lower chamber. Contact experiments were performed with BOEC in contact with fibroblasts or BOEC and fibroblasts alone. Transwell experiments allow for the passage of secreted molecules and do not allow contact to occur. The cells were made hypoxic as described previously, using a hypoxia chamber at $1 \% \mathrm{O}_{2}[7,8,17]$. BOEC were cultured in endothelial basal medium without growth factors (Lonza Cologne $\mathrm{GmbH}$, Walkersville, Md., USA) and fibroblasts were cultured with DMEM (5\% FBS, low-glucose; Invitrogen). In addition, we performed separate experiments using human umbilical-vein endothelial cells (HUVEC; 400,000) alone or in contact with pig fibroblasts $(100,000)$ as outlined above. For the contact experiments, at each time point, the cells were washed with PBS and trypsinized. The cell suspension was incubated with pig CD31 (Abcam, Cambridge, Mass., USA) in PBS with $1 \%$ BSA for $30 \mathrm{~min}$ at $4{ }^{\circ} \mathrm{C}$. The cell suspension was incubated with an anti-mouse magnetic antibody (Milteyni Biotech, San Diego, Calif., USA) in PBS with 1\% BSA. The CD31positive cell population and the BOEC were isolated using magnetic cell sorting and the flow-through fibroblast population was collected for further analysis. 
Table 1. PCR primers used in current study

\begin{tabular}{llll}
\hline Gene & Sequence & Amplicon & Cycles \\
length & & 35 \\
\hline VEGF-A & $\begin{array}{l}\text { 5'-ACCATGCCAAGTGGTCCCAGGC-3' (sense) } \\
\text { 5'-TCCAGACCTTCGTCGTTGCAGC-3' (antisense) }\end{array}$ & 250 & 35 \\
\hline FGF-1 & $5^{\prime}$-AGGGCTGTTCCGACAAGCGC-3' (sense) & 259 & 35 \\
\hline MMP-2 & $5^{\prime}$-TGGCAGGTCCTCTCGGGAGC-3' (antisense) & & 35 \\
\hline TIMP-1 & $5^{\prime}$-CACCATGGCTGAAGGCGAAATCACA-3' (sense) & 250 & 35 \\
& $5^{\prime}$-GATGTCGCCCCCAAAACGGAC-3' (sense) & & 352 \\
\hline
\end{tabular}

Western Blot of $\alpha$-SMA

We assessed the differentiation of fibroblasts into myofibroblasts by performing Western blot analysis for $\alpha$-SMA. The cultured cells were processed and detected using rabbit polyclonal antibody as described previously [11].

\section{Protein Expression Using Proteomic Array}

We assessed the potential mechanism of the proteins responsible for decreasing $\alpha$-SMA expression in the fibroblasts that were in contact with BOEC compared to fibroblasts alone under hypoxia. We used a human angiogenic proteomic array (category No. ARY007; R\&D Biosystems Inc., Minneapolis, Minn., USA) for both the cellular and conditioned media, according to the manufacturer's directions.

\section{RNA Isolation}

The tissue was stored in RNA stabilizing reagent (Qiagen, Gaithersburg, Md., USA) as per the manufacturer's guidelines. To isolate the RNA, the specimens were homogenized and the total RNA was isolated using an RNeasy mini kit (Qiagen) [10, 18].

\section{Real-Time Polymerase Chain Reaction Analysis}

Expression for the gene of interest was determined using realtime polymerase chain reaction (RT-PCR) analysis as described previously [18] (table 1). Please see supplemental methods for full details.

\section{Thymidine Incorporation Assay}

BOEC only, fibroblasts only and fibroblasts in contact with BOEC were seeded in 24-well plates and cultured for 24-72 h in DMEM. After $20 \mathrm{~h}, 1 \mathrm{ci} / \mathrm{mmol}$ of ${ }^{3} \mathrm{H}$-thymidine was added to each well and incubated for $4 \mathrm{~h}$. The cells were washed with chilled PBS, fixed with $100 \%$ cold methanol and then collected for the measurement of trichloroacetic acid-precipitable radioactivity. Experiments were repeated at least 3 times for each time point.

\section{Gelatin Zymography}

Zymography was performed on concentrated conditioned media from the cultured cells of their respective time point as described previously [7].

\section{Cell Migration Assay}

The fibroblasts were loaded with calcein $\mathrm{M}$ dye with $0.1 \%$ pluronic acid in order to observe the differential migration of fibroblasts. The BOEC (approx. 400,000) and pig-vein calcein M-loaded fibroblasts (approx. 100,000) were mixed together in serum-free medium and loaded into the upper chamber of FluoroBlok $8-\mu \mathrm{m}-$ pore transwells (Qiagen), precoated with low growth factor matrigel. The complete media were supplemented under the transwell and incubated for $6 \mathrm{~h}$ at $37^{\circ} \mathrm{C}$. After $6 \mathrm{~h}$, the transwells were washed with PBS and the number of cells was analyzed in relative fluorescence units using a microplate reader.

\section{Statistical Methods}

Data are expressed as mean \pm SEM. Multiple comparisons were performed with 2-way ANOVA followed by the Student $t$ test with the post hoc Bonferroni correction. A significant difference from the control value was indicated by $p<0.05, \mathrm{p}<0.01$, $\mathrm{p}<0.001$ or $\mathrm{p}<0.0001$. JMP version 9 (SAS Institute Inc., Cary, N.C., USA) was used for statistical analyses.

\section{Results}

Expression of $\alpha$-SMA Is Reduced in Fibroblasts in Contact with BOEC Compared to in Fibroblasts Alone under Hypoxic Conditions

We wanted to determine the mechanism by which BOEC reduce the conversion of fibroblasts into myofibroblasts (i.e. a-SMA-positive cells) under hypoxic conditions. This was determined by performing transwell and contact experiments to determine the changes in $\alpha$-SMA using Western blot analysis and confocal microscopy (fig. 1). The experiments were performed in order to determine if the mechanism by which BOEC decreased the conversion of fibroblasts into myofibroblasts was via a para- 


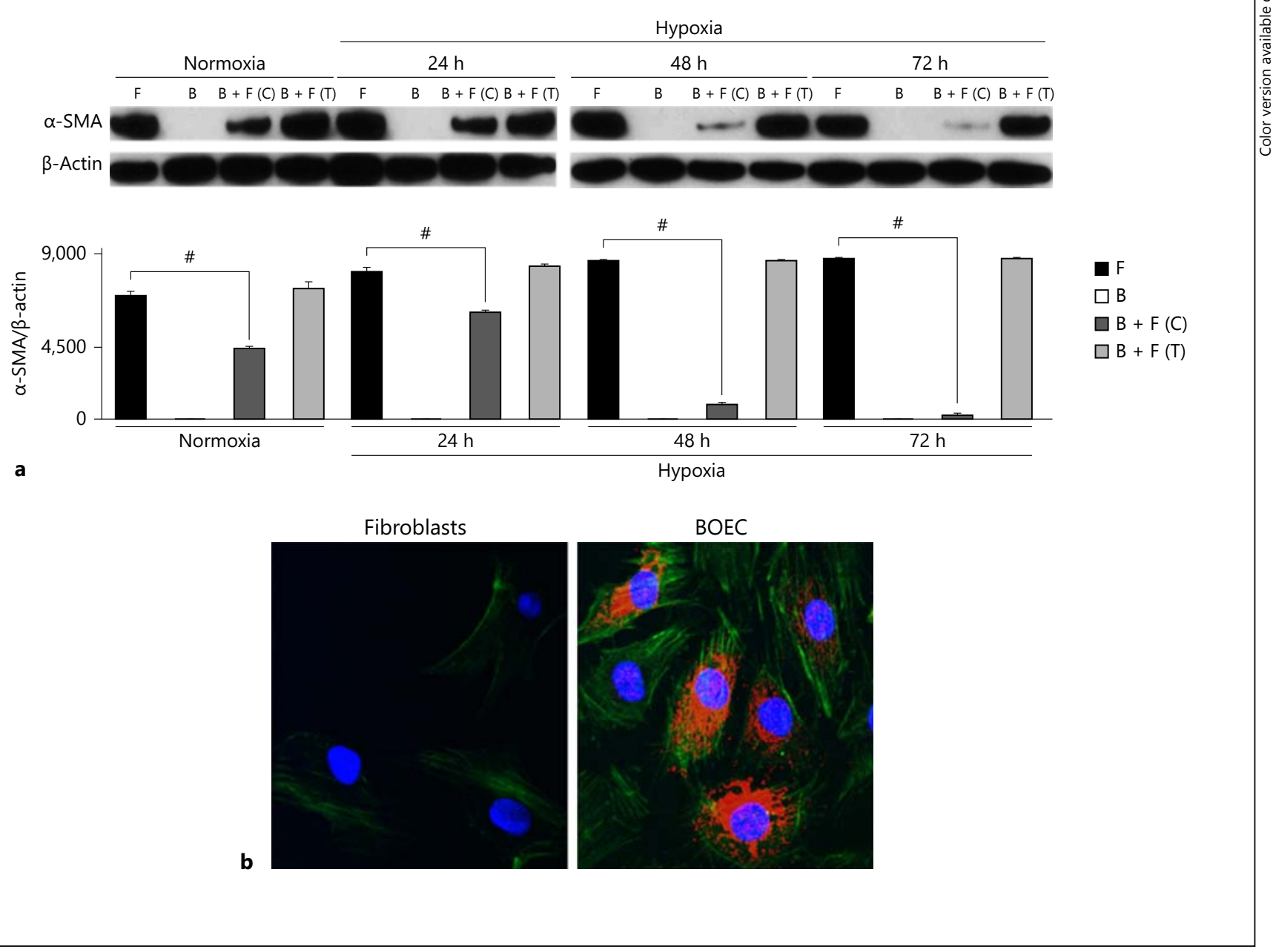

Fig. 1. a Upper panel: fibroblasts in contact with BOEC under hypoxic conditions have reduced $\alpha$-SMA expression compared to fibroblasts alone. Lower panel: pooled Western blot data for contact and transwell experiments at 24,48 and $72 \mathrm{~h}$ of hypoxia for fibroblasts alone (F), BOEC alone (B), BOEC and fibroblasts in contact
$[\mathrm{B}+\mathrm{F}(\mathrm{C})]$ and BOEC and fibroblast in transwells $[\mathrm{B}+\mathrm{F}(\mathrm{T})]$. b Confocal microscopy of a-SMA expression (red). BOEC are phallodin- and von Willebrand factor-positive (green and red, respectively). ${ }^{\#} \mathrm{p}<0.0001$ indicates a significant difference from the control value. crine effect. There was a significant decrease in the average a-SMA expression of fibroblasts when in contact with BOEC compared to fibroblasts alone across all time points [at $24 \mathrm{~h}$ : an average reduction of $28 \%$, fibroblasts alone vs. fibroblasts cultured with BOEC; at $48 \mathrm{~h}$ : an average reduction of $91 \%$, fibroblasts alone vs. fibroblasts cultured with BOEC; at $72 \mathrm{~h}$ : an average reduction of $93 \%$, fibroblasts alone vs. fibroblasts cultured with BOEC; fig. 1a]. However, in the transwell experiments, there was no change in the mean $\alpha$-SMA expression in the fibroblasts in contact with BOEC when compared to the fibroblasts alone.

Normally, fibroblasts are a-SMA-negative whereas BOEC are von Willebrand factor-positive (fig. 1b). Using confocal microscopy, we observed a time-dependent hypoxia-induced decrease in the average $\alpha$-SMA expression in fibroblasts in contact with BOEC compared to in fibroblasts alone (fig. 1c). We also stained fibroblasts in contact with BOEC and fibroblasts alone for $\alpha$-SMA and phylloidin under hypoxic conditions, and demonstrated a reduction in a-SMA/phylloidin-positive expression in the fibroblasts in contact with BOEC compared to fibroblasts alone (online suppl. fig. 1; for all online suppl. material, see www. karger.com/doi/10.1159/000369929). We then determined if other endothelial cells such as HUVEC would have the same effect. Similarly, HUVEC were in contact with pig fibroblasts and determined the $\alpha$-SMA expression in com- 

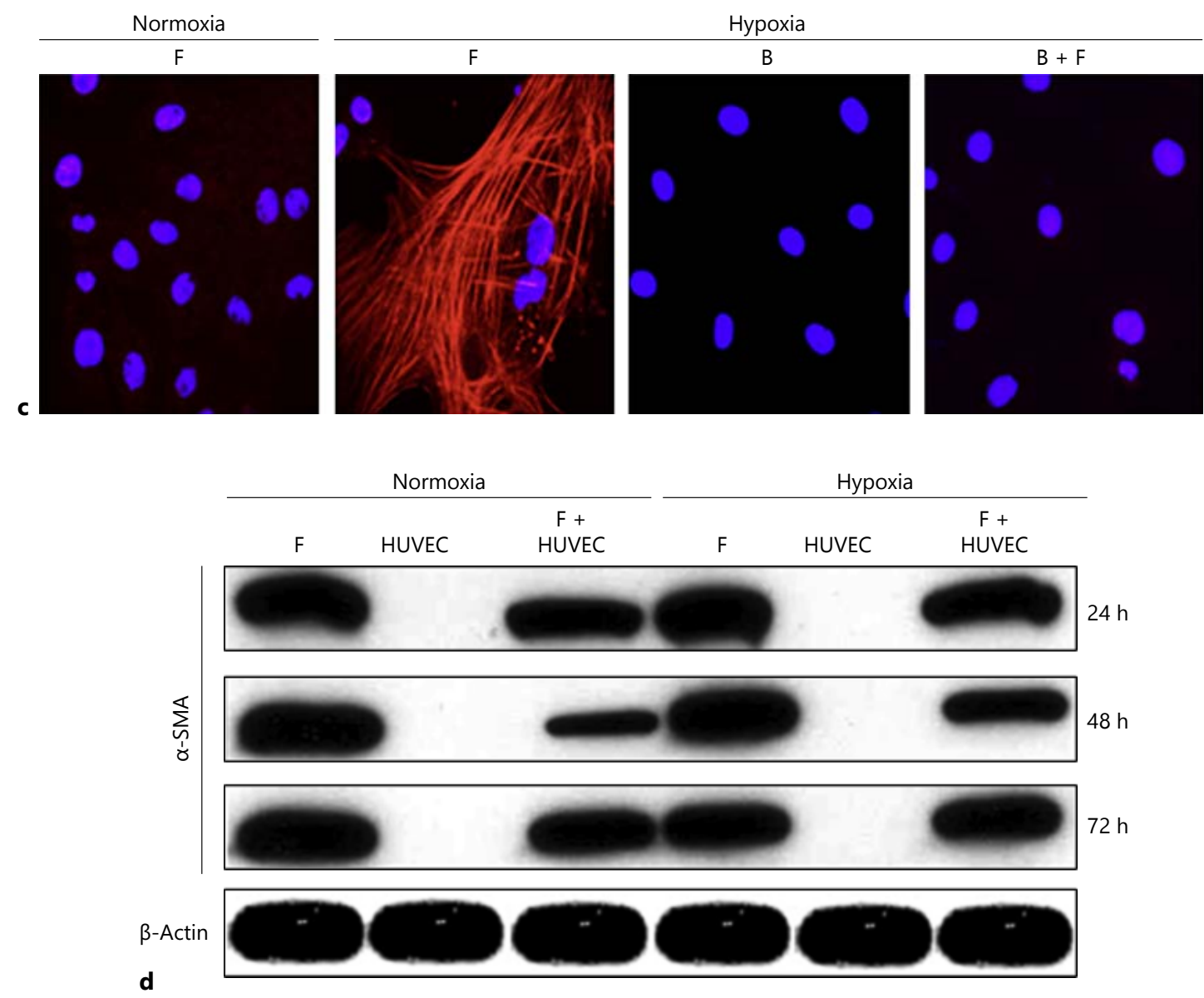

Fig. 1. c Confocal microscopy of fibroblasts alone (F) versus BOEC alone (B) and in contact with fibroblasts $(B+F)$ and exposed to hypoxia at 24-72 h; a-SMA expression is red. d Western blot of fibroblasts (F), HUVEC and fibroblasts and HUVEC in contact (F + HUVEC) at $24-72 \mathrm{~h}$ of normoxia and hypoxia. ${ }^{\#} \mathrm{p}<0.0001$ indicates a significant difference from the control value.

parison to fibroblasts alone under hypoxic conditions. There was no difference in the a-SMA expression in pig fibroblasts in contact with HUVEC when compared to fibroblasts alone under both the transwell and contact conditions (fig. 1d). In order to determine if the effect was perpetuated by viable BOEC, we irradiated BOEC, cultured them in contact with fibroblasts and then compared them to nonirradiated BOEC in contact with fibroblasts, fibroblasts only and BOEC only. They were subjected to 48 and $72 \mathrm{~h}$ of hypoxic conditions. We observed a decrease in the average $\alpha$-SMA expression under hypoxic conditions in fibroblasts in contact with nonirradiated BOEC compared to fibroblasts in contact with irradiated BOEC (fig. 1e).
The Cell Size of Fibroblasts in Contact with BOEC Is Reduced Compared to Fibroblasts Alone

The size of the fibroblasts in contact with BOEC was determined compared to fibroblasts only at different time points under hypoxic conditions. This demonstrated a significant decrease in the average size of the fibroblast in contact with BOEC compared to fibroblasts only [at $24 \mathrm{~h}$ : an average reduction of $28 \%$, fibroblast alone vs. fibroblast in contact with BOEC, respectively $(\mathrm{p}<0.0001)$; at $48 \mathrm{~h}$ : an average reduction of $91 \%$, fibroblast alone vs. fibroblast in contact with BOEC, respectively $(\mathrm{p}<0.0001)$; at $72 \mathrm{~h}$ : an average reduction of $93 \%(\mathrm{p}<0.0001)$; fig. $1 \mathrm{f}]$. 
Fig. 1. e Western blot of BOEC (B), fibroblasts (F), BOEC and fibroblasts in contact (irradiated) $[\mathrm{B}+\mathrm{F}(\mathrm{IR})]$ and $\mathrm{BOEC}$ and fibroblasts in contact $(\mathrm{B}+\mathrm{F})$ under 48 and $72 \mathrm{~h}$ of hypoxia. $\mathrm{f}$ The average cell size of fibroblasts alone (F), BOEC (B) and BOEC and fibroblasts in contact $(\mathrm{B}+\mathrm{F})$ under hypoxia for different lengths of time. Twoway ANOVA, followed by the Student t test with the post hoc Bonferroni correction, was performed. ${ }^{\#} \mathrm{p}<0.0001$ indicates a significant difference from the control value.

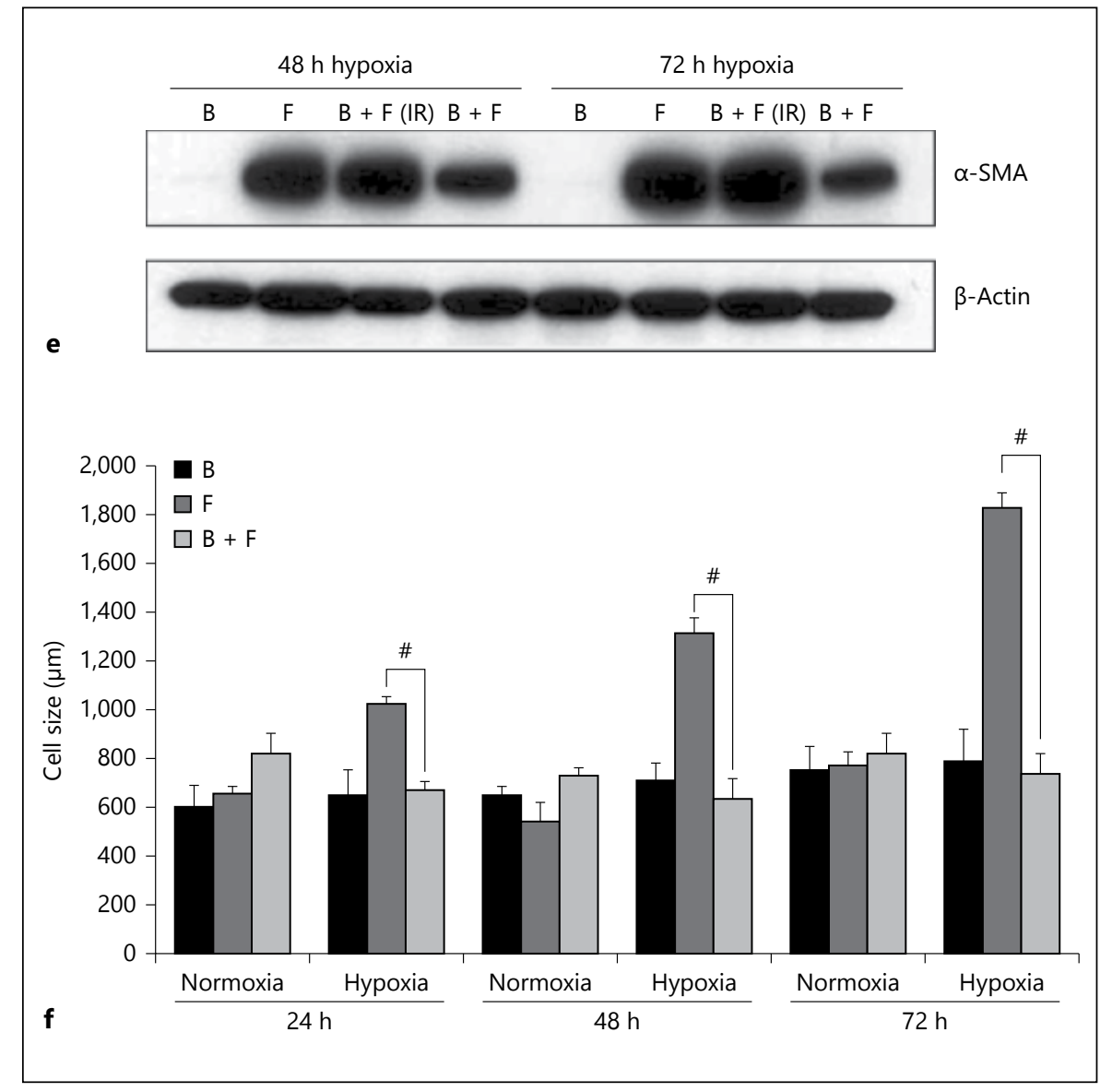

\section{Identification of the Potential Mechanism Using a}

Proteomic Array

In order to determine the mechanism that may be responsible for the decreased expression of a-SMA, an angiogenic protein array was used along with Ingenuity System software for pathway analysis. This demonstrated that there was a decrease in several proteins including VEGF-A, FGF-1, PDGF, MMP-2 and tissue inhibitor of matrix metalloproteinase 1 (TIMP-1).

Gene Expression of VEGF-A, PDGF, FGF-1, MMP-2 and TIMP-1 Is Reduced in Fibroblasts in Contact with BOEC Compared to Fibroblasts Alone under Hypoxic Conditions

Next, we determined the gene expression of VEGF-A, PDGF, FGF-1, MMP-2 and TIMP-1 in fibroblasts in contact with BOEC when compared to fibroblasts alone under hypoxic conditions using RT-PCR analysis (fig. 2). All of these genes have been implicated in $\mathrm{VNH}$ formation associated with hemodialysis vascular access failure. We observed that in fibroblasts in contact with BOEC when compared to fibroblasts alone, there was a significant decrease in the mean VEGF-A expression at $24 \mathrm{~h}$ (an average reduction of $61 \%, \mathrm{p}<0.0001)$ and $72 \mathrm{~h}$ (an average reduction of $72 \%, \mathrm{p}<0.0001)$. In addition, we observed a significant decrease in the average gene expression of PDGF, FGF-1, MMP-2 and TIMP-1 in fibroblasts in contact with BOEC when compared to fibroblasts alone at 24 h [PDGF: an average reduction of $95 \%(\mathrm{p}<0.0001)$, FGF-1: an average reduction of $93 \%(\mathrm{p}<0.0001)$, MMP2: an average reduction of $95 \%(\mathrm{p}<0.0001)$ and TIMP-1: an average reduction of $34 \%(\mathrm{p}<0.01)$ ] and $72 \mathrm{~h}$ [PDGF: an average reduction of $98 \%(\mathrm{p}<0.0001)$, FGF- 1 : an average reduction of $87 \%(\mathrm{p}<0.0001)$, MMP-2: an average reduction of $90 \%(\mathrm{p}<0.0001)$ and TIMP-1: an average reduction of $21 \%(\mathrm{p}<0.001)$ ].

\section{Migration Is Decreased in Fibroblasts in Contact with BOEC Compared to Fibroblasts under Hypoxic Conditions}

Since several of the genes implicated in cellular migration were reduced by RT-PCR analysis, we hypothesized 
Fig. 2. Gene expression of VEGF-A (a), PDGF (b), FGF-1 (c), MMP-2 (d) and TIMP-1 (e) in BOEC and fibroblasts in contact $(\mathrm{B}+\mathrm{F})$ compared to fibroblast alone (F) and BOEC alone (B) under hypoxic conditions. Expression was decreased in fibroblasts in contact with BOEC at 24 and $72 \mathrm{~h}$ of hypoxia. Two-way ANOVA followed by the Student t test with the post hoc Bonferroni correction was performed. ${ }^{* *} \mathrm{p}<0.01,{ }^{* * *} \mathrm{p}<0.001,{ }^{*} \mathrm{p}<$ 0.0001 indicate a significant difference from the control value.
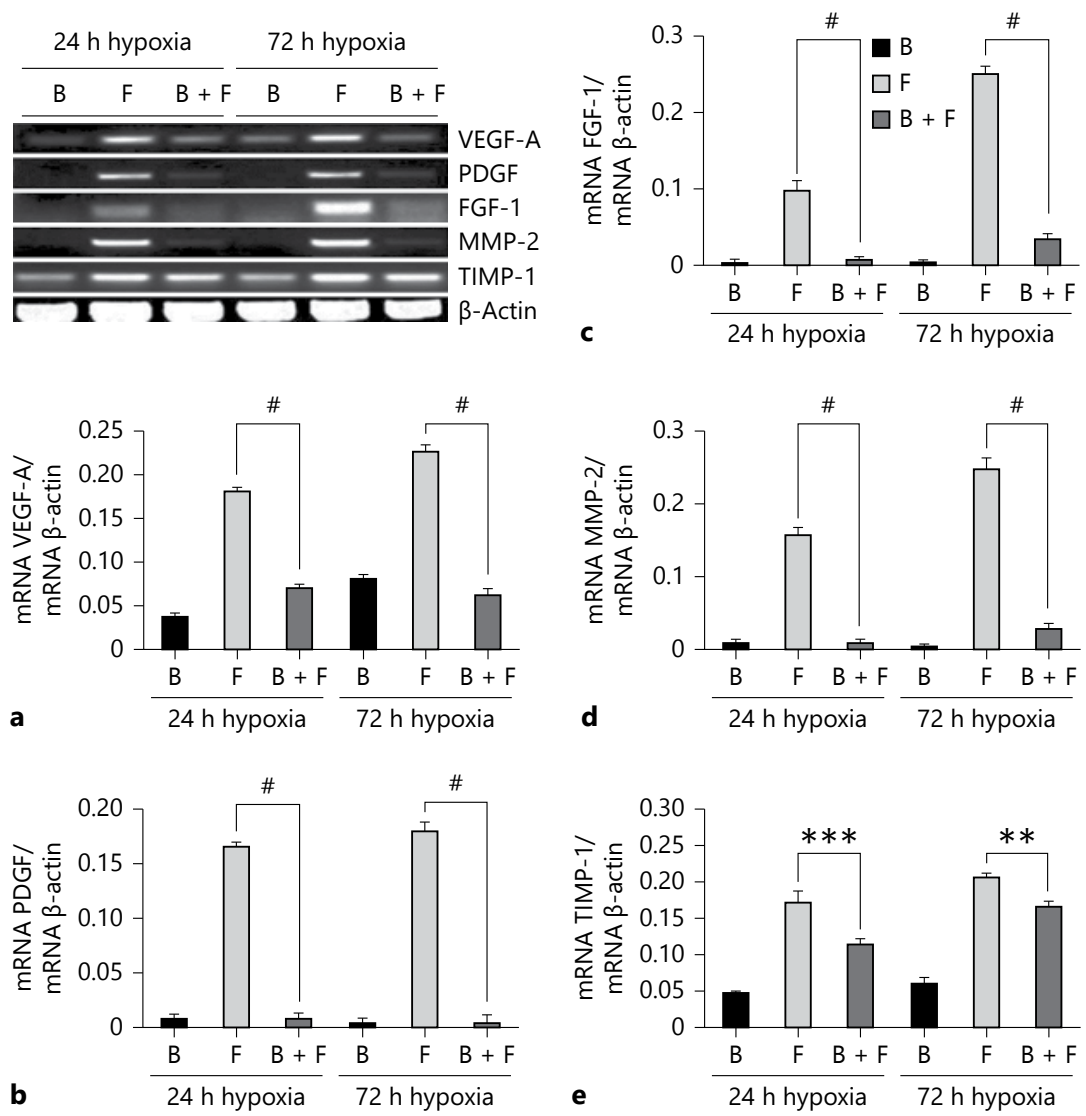

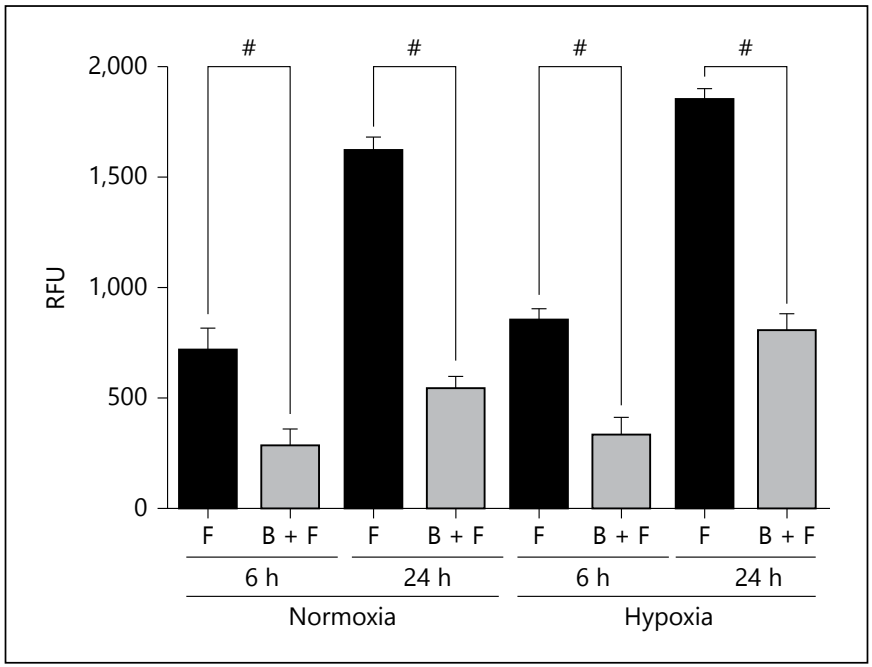

Fig. 3. Differential migration of BOEC and fibroblasts in contact $(\mathrm{B}+\mathrm{F})$, fibroblasts alone $(\mathrm{F})$ and BOEC alone $(\mathrm{B})$ under hypoxia for different lengths of time. Two-way ANOVA followed by the Student $t$ test with the post hoc Bonferroni correction was performed. ${ }^{\#} \mathrm{p}<0.0001$ indicates a significant difference from the control value. $\mathrm{RFU}=$ Relative fluorescence units. that there would be a decrease in cellular migration in fibroblasts in contact with BOEC when compared to fibroblasts alone. We observed a significant decrease in the migration of fibroblasts in contact with BOEC when compared to fibroblasts alone at both $6 \mathrm{~h}$ and $24 \mathrm{~h}$ of hypoxia [at $6 \mathrm{~h}$ : an average reduction of $28 \%$, fibroblasts alone vs. fibroblasts in contact with BOEC, respectively ( $p<0.0001$ ); at $24 \mathrm{~h}$ : an average reduction of $91 \%$, fibroblasts alone vs. fibroblasts in contact with BOEC, respectively ( $<<0.0001$; fig. 3 )].

\section{Proliferation Is Not Decreased in Fibroblasts in Contact with BOEC Compared to Fibroblasts under Hypoxic Conditions}

Finally, we determined cellular proliferation changes in fibroblasts in contact with BOEC when compared to fibroblasts alone. We observed a significant increase in proliferation under hypoxia of fibroblasts in contact with BOEC when compared to fibroblasts alone at 24 to $72 \mathrm{~h}$ of hypoxia ( $\mathrm{p}<0.0001$; fig. 4$)$. 
Fig. 4. Proliferation of fibroblasts by ${ }^{3} \mathrm{H}-$ thymidine incorporation in BOEC and fibroblasts in contact $(B+F)$, fibroblasts alone $(\mathrm{F})$ and BOEC alone (B) under hypoxia for different lengths of time. Twoway ANOVA followed by the Student t test with the post hoc Bonferroni correction was performed. ${ }^{* *} \mathrm{p}<0.01,{ }^{* * *} \mathrm{p}<0.0001$, \# $\mathrm{p}<0.0001$ indicate a significant difference from the control value.

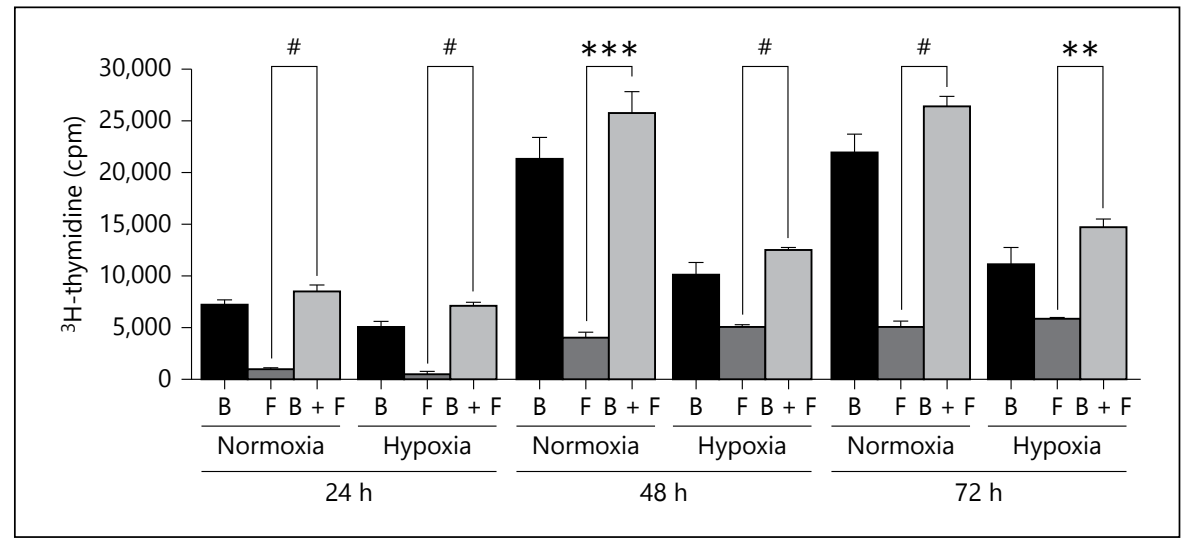

Fig. 5. Zymographic analysis of BOEC alone (B), fibroblasts alone (F), and BOEC and fibroblasts in contact $(\mathrm{B}+\mathrm{F})$ under hypoxia for different lengths of time. There were changes in pro- and active-MMP-9 expression in BOEC and fibroblasts in contact $(\mathrm{B}+$ F) compared to fibroblasts alone $(\mathrm{F})$.

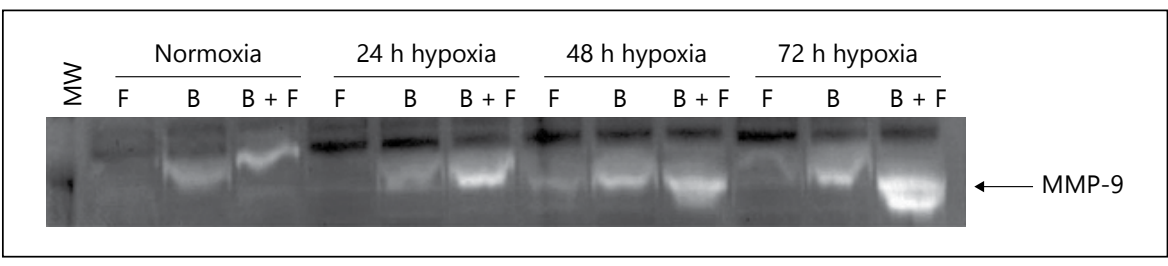

MMP-9 Activity Is Increased in Fibroblasts in Contact with BOEC Compared to Fibroblasts Alone under Hypoxic Conditions

In our experiments with adventitial transplantation of BOEC to AV hemodialysis grafts, a significant increase in MMP-9 activity by zymography was observed and MMP9 has been shown to cause an increase in cellular proliferation. We determined the MMP-9 expression using zymography in fibroblasts in contact with BOEC and compared to fibroblast alone. We observed an increase in both pro-MMP-9 and active MMP-9 in fibroblasts in contact with BOEC when compared to fibroblast alone (fig. 5). In addition, no significant change in MMP-2 was observed for the same experiments (see online suppl. fig. 2). These results suggest that the increase in MMP-9 activity may be responsible for the increase in proliferation.

\section{Discussion}

In this study, we determined the mechanism via which BOEC may decrease the conversion of fibroblasts to myofibroblasts mediated under hypoxic conditions. Previously, we described that BOEC transplantation to the adventitia of the outflow vein of porcine arteriovenous hemodialysis grafts results in a reduction of $\mathrm{VNH}$ via a reduction in HIF$1 \alpha$ expression [6]. We wanted to determine how BOEC re- duce $\mathrm{VNH}$, and we hypothesized that they influence the conversion of fibroblasts to myofibroblasts via either a paracrine or autocrine mechanism. Here, we demonstrate that contact needs to occur between the BOEC and the fibroblasts. Under hypoxic conditions, when fibroblasts are in contact with BOEC, there is a significant reduction in the migration and size of cells, and in the mean $\alpha$-SMA expression. In addition, we observed a significant decrease in the expression of several genes including VEGF-A, PDGF, FGF-1, MMP-2 and TIMP-1 (fig. 6). These findings have many implications for hemodialysis vascular failure as all of these cytokines and TIMP-1 have been increased in experimental animal models of hemodialysis vascular access failure and specimens removed from patients [9-12].

Previous research has shown that hypoxia can cause an increase in the conversion of fibroblasts to myofibroblasts [7]. Our laboratory has shown that there is an increase in HIF-1 $\alpha$ level in specimens removed from experimental animal models and clinical specimens removed from patients with failed hemodialysis vascular access $[12,19]$. Furthermore, adventitial fibroblasts have been shown to contribute to smooth-muscle cells comprising $\mathrm{VNH}$ associated with hemodialysis graft failure $[13,20,21]$. We hypothesize that disruption of the vasa vasorum at the time of vascular access placement can cause an increase in local vessel hypoxia that leads to the activation of adventitial fibroblasts that convert to myo- 
Fig. 6. Schematic of proposed interaction by BOEC according to changes in hypoxic conditions.



fibroblasts. Furthermore, in our laboratory, we demonstrated that adventitial transplantation of BOEC to the outflow vein causes a decrease in HIF-1a in the transplanted BOEC vessels when compared to controls [6].

We assessed the mechanism by which BOEC might influence the conversion of fibroblasts into myofibroblasts by means of a contact experiment between the BOEC and fibroblasts and a transwell experiment. In the transwell experiment, we observed that there was no reduction in the conversion of fibroblasts to myofibroblasts when cultured with BOEC compared to fibroblasts under hypoxic conditions. However, when fibroblasts were in contact with BOEC and then separated using magnetic beads, there was a significant reduction in $\alpha$-SMA expression in these fibroblasts compared to fibroblasts alone. This experiment implies that such contact is necessary for the reduction in a-SMA to occur. Furthermore, we demonstrated that the results would not be the same if the fibroblasts were in contact with HUVEC or irradiated BOEC. These observations are consistent with experimental animal models of wound-healing [22], where it was found that the BOEC needed to be in contact with the fibroblasts in order for the beneficial effects to occur.

To determine the potential mechanism responsible for these observations, we performed a protein array that identified several proteins that were decreased including VEGFA, PDGF, FGF-1 and MMP-2. Interestingly, each of these has been found to be elevated and to be associated with VNH $[9-13,23,24]$. We determined the messenger RNA levels for each of these genes using RT-PCR, and found them to all be reduced in fibroblasts in contact with BOEC compared to fibroblasts alone. These results are consistent with other experiments which have demonstrated that BOEC under hypoxic conditions will secrete proangiogenic genes that have a paracrine effect. This study demonstrates, with an in vitro model, that there is a decrease in proangiogenic gene expression in other cells, notably fibroblasts, which is mediated through contact [25]. This decrease in proangiogenic genes is most likely responsible for the decrease in $\alpha$-SMA expression. We have recently shown that silencing VEGF-A in fibroblasts and then subjecting them to hypoxia decreased $\alpha$-SMA expression compared to in controls [8]. This result is consistent with other studies using experimental models of skin healing [22].

Finally, we examined the proliferation and migration of fibroblasts in contact with BOEC We observed that migration was significantly reduced in fibroblasts in contact with BOEC when compared to fibroblasts alone. This result is consistent with observations where VEGF-A reduction has been shown to result in a decrease in proliferation [8]. However, proliferation was increased, which most likely reflects the increase in MMP-9 activity that we observed. These results are consistent with our in vivo experiments, where we observed increased MMP-9 activity in BOEC transplanted vessels compared to controls [26].

There are several limitations to this study. The results of our research need to be validated in a human cell-line model with BOEC from both healthy subjects and patients with chronic kidney disease. This would reveal the benefits of BOEC as a cell therapy in patients. In addition, the in vitro experiments do not simulate the in vivo experiments and these need to be conducted with the cells using a xenotransplant model. Finally, the durability of the observations needs to be determined in chronic animal models.

In summary, our results indicate that BOEC when in contact with fibroblasts reduce the conversion of fibroblasts to myofibroblasts, mediated in part through a reduction of several proangiogenic genes including VEGF-A, PDGF, FGF-1 and MMP-2 with a reduction in migration. This has implications for hemodialysis vascular access failure, because it is hypothesized that $\mathrm{VNH}$ is caused by many of the cytokines that were found to be reduced. 


\section{Acknowledgements}

This work was funded by the National Heart, Lung, and Blood Institute (HL098967 to S.M.).

\section{Disclosure Statement}

No disclosures.

\section{References}

1 Collins AJ, Kasiske B, Herzog C, Chen SC, Everson S, Constantini E, Grimm R, McBean M, Xue J, Chavers B, Matas A, Manning W, Louis T, Pan W, Liu J, Li S, Roberts T, Dalleska F, Snyder J, Ebben J, Frazier E, Sheets D, Johnson R, Dunning S, Berrini D, Guo H, Solid C, Arko C, Daniels F, Wang X, Forrest B, Gilbertson D, St Peter W, Frederick P, Eggers P, Agodoa L: Excerpts from the United States Renal Data System 2003 annual data report: atlas of end-stage renal disease in the United States. Am J Kidney Dis 2003;42:A5-A7.

$\checkmark 2$ Rooijens PPGM, Tordoir JHM, Stijnen T, Burgmans JPJ, Smet de AAEA, Yo TI: Radiocephalic wrist arteriovenous fistula for hemodialysis: meta-analysis indicates a high primary failure rate. Eur J Vasc Endovasc Surg 2004; 28:583-589.

-3 Westerweel PE, Hoefer IE, Blankestijn PJ, de Bree P, Groeneveld D, van Oostrom O, Braam B, Koomans HA, Verhaar MC: End-stage renal disease causes an imbalance between endothelial and smooth muscle progenitor cells. Am J Physiol Renal Physiol 2007;292:F1132-F1140.

-4 Baigent C, Burbury K, Wheeler D: Premature cardiovascular disease in chronic renal failure. Lancet 2000;356:147-152.

$\checkmark 5$ Gulati R, Jevremovic D, Peterson TE, Witt TA, Kleppe LS, Mueske CS, Lerman A, Vile RG, Simari RD: Autologous culture-modified mononuclear cells confer vascular protection after arterial injury. Circulation 2003;108: 1520-1526.

6 Hughes D, Fu AA, Puggioni A, Glockner JF, Anwer B, McGuire AM, Mukhopadhyay D, Misra S: Adventitial transplantation of blood outgrowth endothelial cells in porcine haemodialysis grafts alleviates hypoxia and decreases neointimal proliferation through a matrix metalloproteinase-9-mediated pathway - a pilot study. Nephrol Dial Transplant 2009;24:85-96.

7 Misra S, Fu AA, Misra KD, Shergill UM, Leof EB, Mukhopadhyay D: Hypoxia-induced phenotypic switch of fibroblasts to myofibroblasts through a matrix metalloproteinase $2 /$ tissue inhibitor of metalloproteinase-mediated pathway: implications for venous neointimal hyperplasia in hemodialysis access. J Vasc Interv Radiol 2010;21:896-902.

8 Yang B, Janardhanan R, Vohra P, Greene EL, Bhattacharya S, Withers S, Roy B, Nieves Torres E, Mandrekar J, Leof EB, Mukhopadhyay D, Misra S: Adventitial transduction of lentivirus-shRNA-VEGF-A treatment in arteriovenous fistula reduces venous stenosis formation. Kidney Int 2014;85:289-306.
$\$ 9$ Roy-Chaudhury P, Kelly BS, Miller MA, Reaves A, Armstrong J, Nanayakkara N, Heffelfinger SC: Venous neointimal hyperplasia in polytetrafluoroethylene dialysis grafts. Kidney Int 2001;59:2325-2334.

10 Misra S, Shergill U, Yang B, Janardhanan R, Misra KD: Increased expression of HIF-1alpha, VEGF-A and its receptors, MMP-2, TIMP-1, and ADAMTS- 1 at the venous stenosis of arteriovenous fistula in a mouse model with renal insufficiency. J Vasc Interv Radiol 2010;21:1255-1261.

11 Misra S, Fu AA, Puggioni A, Karimi KM, Mandrekar JN, Glockner JF, Juncos LA, Anwer B, McGuire AM, Mukhopadhyay D: Increased shear stress with up regulation of VEGF-A and its receptors and MMP-2, MMP-9, and TIMP-1 in venous stenosis of hemodialysis grafts. Am J Physiol Heart Circ Physiol 2008;294:H2219-H2230.

12 Misra S, Fu AA, Rajan DK, Juncos LA, McKusick MA, Bjarnason H, Mukhopadhyay D: Expression of hypoxia inducible factor-1 alpha, macrophage migration inhibition factor, matrix metalloproteinase- 2 and -9 , and their inhibitors in hemodialysis grafts and arteriovenous fistulas. J Vasc Interv Radiol 2008;19: 252-259.

13 Misra S, Doherty MG, Woodrum D, Homburger J, Mandrekar JN, Elkouri S, Sabater EA, Bjarnason H, Fu AA, Glockner JF, Greene EL, Mukhopadhyay D: Adventitial remodeling with increased matrix metalloproteinase-2 activity in a porcine arteriovenous polytetrafluoroethylene grafts. Kidney Int 2005;68:2890-2900.

14 Weiss MF, Scivittaro V, Anderson JM: Oxidative stress and increased expression of growth factors in lesions of failed hemodialysis access. Am J Kidney Dis 2001;37:970980.

$\checkmark 15$ Lin Y, Weisdorf DJ, Solovey A, Hebbel RP: Origins of circulating endothelial cells and endothelial outgrowth from blood. J Clin Invest 2000;105:71-77.

16 Das M, Dempsey EC, Reeves JT, Stenmark KR: Selective expansion of fibroblast subpopulations from pulmonary artery adventitia in response to hypoxia. Am J Physiol Lung Cell Mol Physiol 2002;282:L976-L986.
17 Janardhanan R, Yang B, Vohra P, Roy B, Withers S, Bhattacharya S, Mandrekar J, Kong H, Leof EB, Mukhopadhyay D, Misra S: Simvastatin reduces venous stenosis formation in a murine hemodialysis vascular access model. Kidney Int 2013;84:338-352.

18 Yang B, Shergill U, Fu AA, Knudsen B, Misra $S$ : The mouse arteriovenous fistula model. J Vasc Interv Radiol 2009;20:946-950.

19 Misra S, Fu A, Pugionni A, Glockner J, Rajan $\mathrm{D}$, McKusick M, Bjarnason H, Mukhopadhyay $\mathrm{D}$ : Increased expression of hypoxia inducible factor-1a in a porcine model of chronic renal insufficiency with arteriovenous polytetrafluoroethylene grafts. J Vasc Interv Radiol 2008; 19:260-265.

20 Li L, Terry CM, Blumenthal DK, Kuji T, Masaki T, Kwan BCH, Zhuplatov I, Leypoldt JK, Cheung AK: Cellular and morphological changes during neointimal hyperplasia development in a porcine arteriovenous graft model. Nephrol Dial Transplant 2007;22:3139-3146.

-21 Wang Y, Krishnamoorthy M, Banerjee R, Zhang J, Rudich S, Holland C, Arend L, RoyChaudhury P: Venous stenosis in a pig arteriovenous fistula model anatomy, mechanisms and cellular phenotypes. Nephrol Dial Transplant 2007;22:3139-3146.

22 Hendrickx B, Verdonck K, Van den Berge S, Dickens S, Eriksson E, Vranckx JJ, Luttun A: Integration of blood outgrowth endothelial cells in dermal fibroblast sheets promotes full thickness wound healing. Stem Cells 2010;28: 1165-1177.

23 Misra S, Misra KD, Glockner JF: Vascular endothelial growth factor-a, matrix metalloproteinase-1, and macrophage migration inhibition factor changes in the porcine remnant kidney model: evaluation by magnetic resonance imaging. J Vasc Interv Radiol 2010;21: 1071-1077.

24 Rotmans JI, Velema E, Verhagen HJ, Blankensteijn JD, de Kleijn DP, Stroes ES, Pasterkamp G: Matrix metalloproteinase inhibition reduces intimal hyperplasia in a porcine arteriovenous-graft model. J Vasc Surg 2004;39: 432-439.

25 Sieveking DP, Buckle A, Celermajer DS, Ng MK: Strikingly different angiogenic properties of endothelial progenitor cell subpopulations: insights from a novel human angiogenesis assay. J Am Coll Cardiol 2008;51:660-668.

-26 Legrand C, Polette M, Tournier JM, de Bentzmann S, Huet E, Monteau M, Birembaut P: uPA/plasmin system-mediated MMP-9 activation is implicated in bronchial epithelial cell migration. Exp Cell Res 2001;264:326-336. 